\title{
NON-ACCIDENTAL INJURY
}

Dr Nigel Speight; FRCP, is consultant paediatrician to Durham and North West Durham health authorities. The ABC of Clinical Genetics will continue next week. The third article in this series will appear on 15 April.

\begin{abstract}
Nigel Speight
Child abuse is the difference between a hand on the bottom and a fist in the face.

HENRY KEMPE

Difficulty of diagnosis

The diagnosis of physical abuse (non-accidental injury) is a difficult intellectual and emotional exercise. It is one of the most difficult subjects in clinical work, needing time, experience, and emotional energy. The biggest barrier to diagnosis is the existence of emotional blocks in the minds of professionals. These can be so powerful that they prevent the diagnosis even being considered in quite obvious cases (see, for example, case 1).

All those working with children should be warned that their overwhelming impulse on confronting their first case will be to want to cover it up.

The most important step in diagnosing
\end{abstract}

non-accidental injury is to force yourself to think of it in the first place.

\section{Importance of diagnosis}

Non-accidental injury is one of the most important diagnoses in clinical paediatrics as it can so vitally influence a child's future life. At worst it is a matter of life and death for the child, and short of death there may still be possible brain damage or handicap. Though high risk cases are currently in a minority, the diagnosis remains crucially important in every case. This is because non-accidental injury is often a marker for emotional abuse and deprivation that can cause progressive and possibly permanent damage to a child's developing personality. This principle is especially relevant in children of school age. In such children the risk of death may be extremely small, yet non-accidental injury in older children is almost inevitably associated with a longstanding disturbance of the parent-child relationship. For this reason non-accidental injury should never be dismissed as "overchastisement."

Non-accidental injury is not a full diagnosis, it is merely a symptom of disordered parenting.

The aim of intervention is to diagnose and cure (if possible) the disordered parenting. Simply to aim at preventing death is a lowly ambition.

In some cases the occurrence of physical abuse may provide an opportunity for intervention. If this opportunity is missed there may be no further opportunity for many years.

\section{Diagnostic features}

There are no hard and fast rules and no easy answers for diagnosis. The following list constitutes seven classic "pointers" to the diagnosis. None of them is diagnostic, neither does the absence of any of them exclude the diagnosis of non-accidental injury.

(1) There is a delay in seeking medical help (or medical help is not sought at all).

(2) The story of the "accident" is vague, is lacking in detail, and may vary with each telling and from person to person. (Innocent accidents tend to have vivid accounts that ring true.)

(3) The account of the accident is not compatible with the injury observed.

(4) The parents' affect is abnormal. Normal parents are full of creative anxiety for the child. Abusing parents tend to be more preoccupied with their own problems - for example, how they can return home as soon as possible.

(5) The parents' behaviour gives cause for
The next morning she told a nurse, "Daddy told Mummy he would never do i again." On further questioning the father became aggressive and had to be restrained by police from abducting the child after attempting to remove her Gallow's traction. 


\section{Case 2: The importance of the child's appearance}

These pictures show the "before and after" effect when severe deprivation is reversed. This 3 year old boy was in fact never subjected to non-accidental injury, but many children in cases of non-accidental injury may have the same appearance. He was admitted after a genuine accident and noted to be extremely deprived. The bond between him and his mother was non-existent. (He had been admitted two years before with non-organic failure to thrive and retardation but allowed to lapse from medical follow up with no referral to social services.) In the ward he showed noticeable affection-seeking behaviour and flourished. The follow up photograph was taken six months later while he was in foster care.
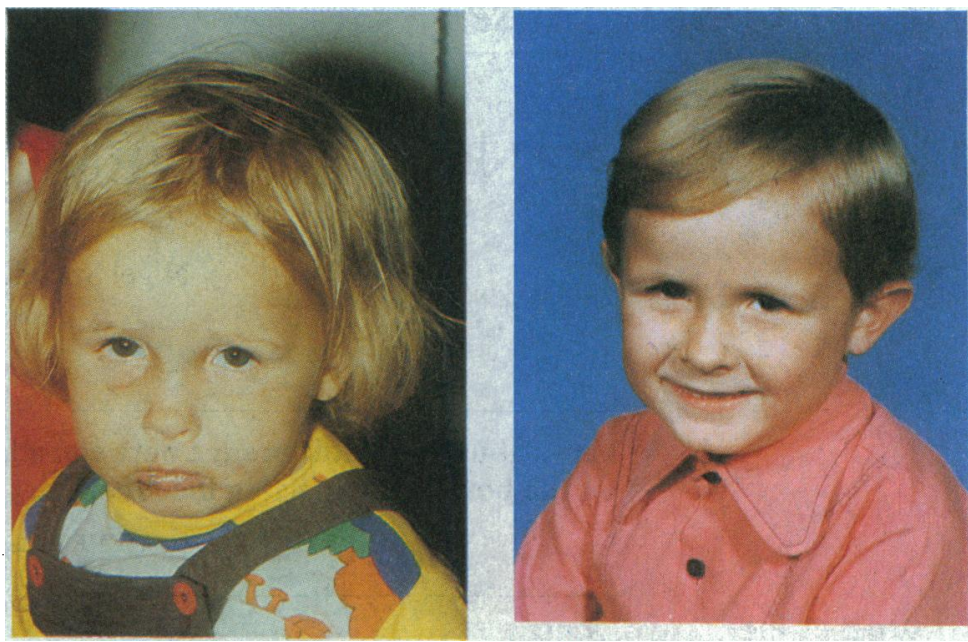

concern - for example, they soon become hostile, they rebut accusations that have not been made, and they leave before the consultant arrives.

(6) The child's appearance and his interaction with his parents is abnormal. He may look sad, withdrawn, or frightened (case 2). There may be visible evidence of a failure to thrive. Full blown frozen watchfulness is a late stage and results from repetitive physical and emotional abuse over a period of time. The absence of frozen watchfulness does not exclude the diagnosis of non-accidental injury.

(7) The child may say something (case 1). Always make a point of interviewing the child (if old enough) in a safe place in private. This is one of the virtues of admission to hospital.

Interviewing the child as an outpatient may fail to let the child open up as he is expecting to be returned to the custody of the abusing parent in the near future.

\section{Characteristic patterns of injury}

Some forms of injury are by their nature virtually diagnostic of non-accidental injury. These include finger tip bruising, especially when it is multiple (cases 3 and 4); cigarette burns; lash marks (case 5); retinal and subdural haemorrhages (figure), which can occur after a shaking injury without other evidence of bruising or fracture; and a torn frenulum.

\section{How to approach a case of suspected non-accidental injury}

Firstly, approach the parents as you would in any other clinical case. Introduce yourself, shake hands, and proceed logically through the history, examination, provisional diagnosis, and decision making.

Secondly, do not jump to conclusions one way or the other. Try to proceed slowly and deliberately, keeping your mind open as long as possible. Take a provisional social history to form a picture of the family's background and how likely it would be for abuse to occur there.

\section{Referral pathway}

The diagnosis of non-accidental injury is a two tier exercise. The doctor on the front line (general practitioner, senior house officer in the accident and emergency department, or school doctor) is responsible for identifying children with suspected physical abuse and referring them to a consultant paediatrician for definitive diagnosis.

\section{Role of front line doctors}

Doctors on the front line must realise and accept the limits of their responsibility. They do not have to make a definitive diagnosis and should have a comparatively low threshold of referral. It should be accepted that some children

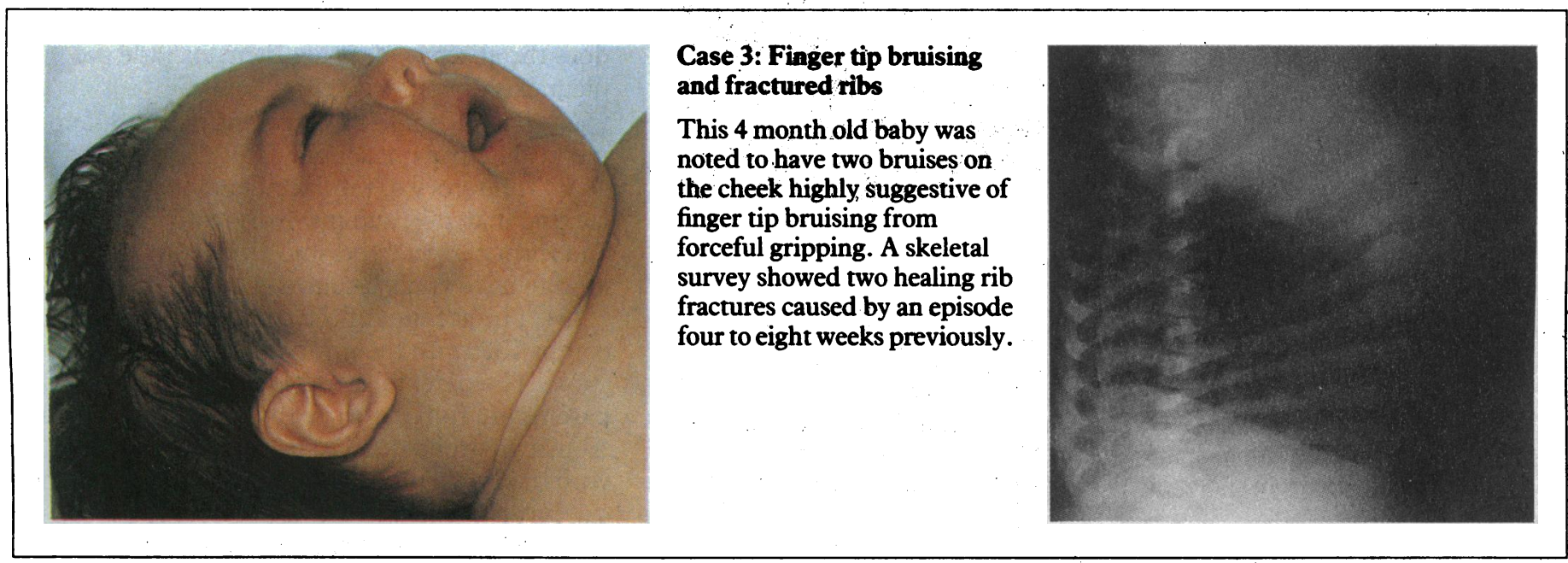




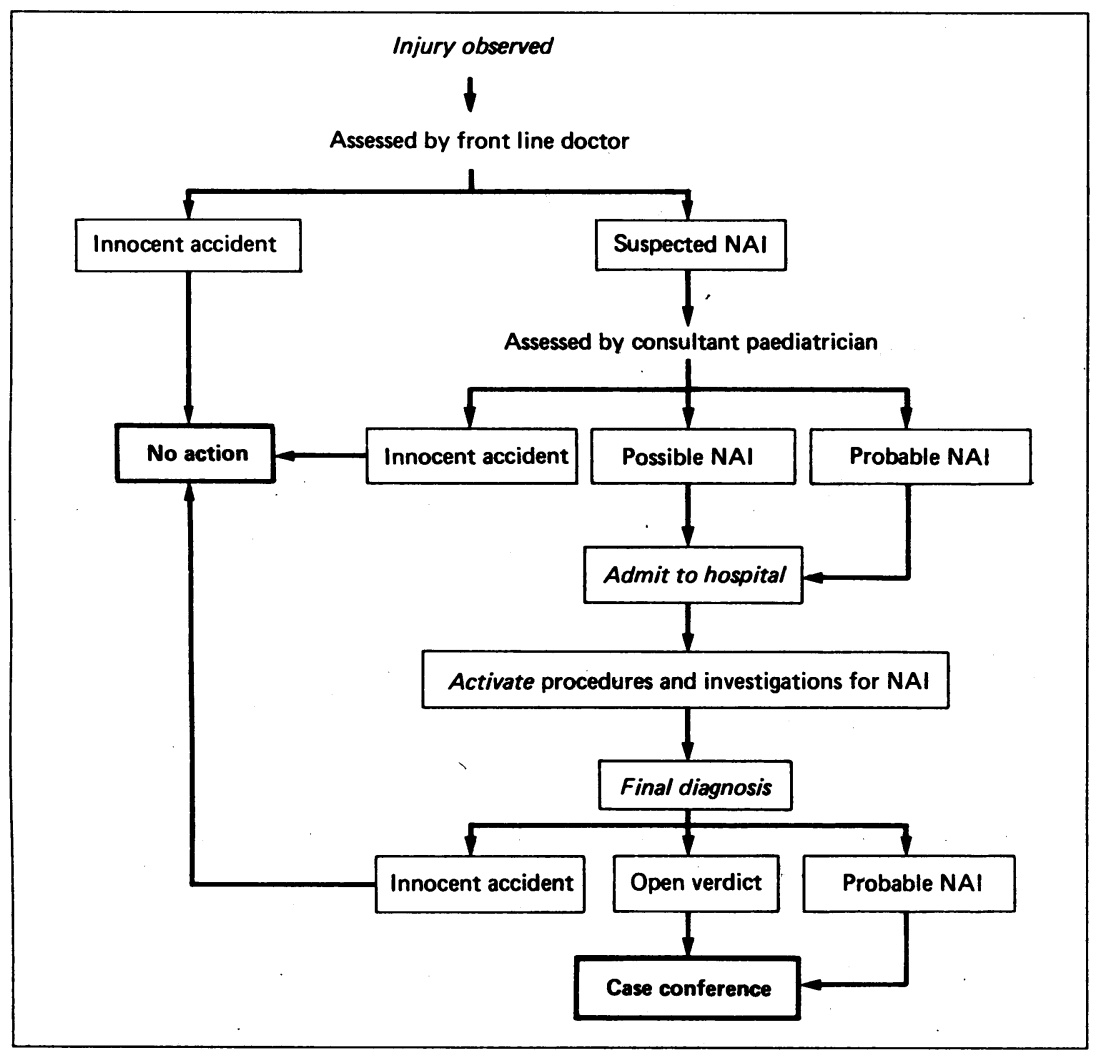

Flow chart for referral of cases of suspected non-accidental injury (NAI)

\section{Case 4: Finger tip bruising}

This 2 year old boy was admitted with a fractured tibia, bruising to the face, and classic finger tip bruising over the right scapula.

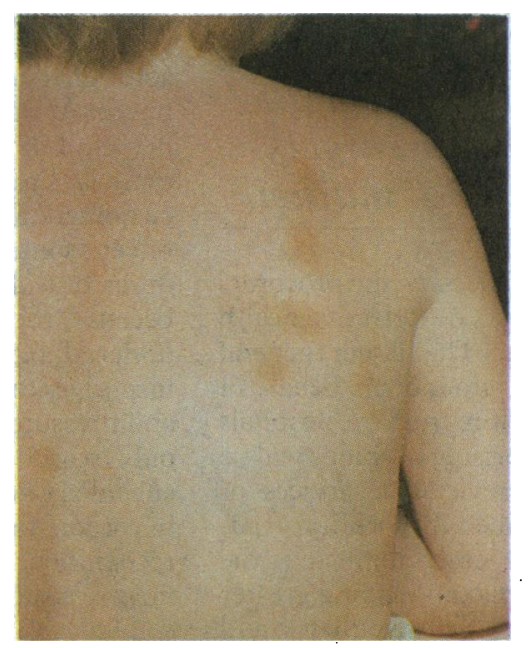

\section{Case 5: Lash marks}

This 10 year old girl was whipped with a belt by her depressed father. The linear nature of the marks is seen, together with a tramlining effect, in which the point of impact is white and the adjoining skin shows bruising caused by capillaries that have been broken by blood being forced into them.

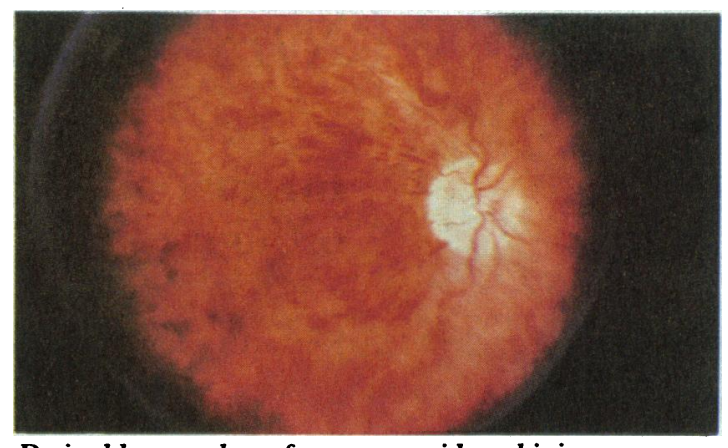

Retinal haemorrhage from non-accidental injury

with innocent injuries will be referred. If all of the children referred turn out to have been physically abused the threshold for referral is almost certainly too high.

Front line doctors should not feel guilt about referring children with suspected non-accidental injury to a paediatrician. They are not accusing either parent; they are simply asking for a second opinion on an important and difficult diagnosis. The fact that the child is being referred because of suspected non-accidental injury should be conveyed to the parents in a neutral and matter of fact way. It is not in the interests of children or parents for child abuse to be covered up. To do so leaves the parents at greater risk of inflicting more severe injuries next time, being imprisoned for causing more severe injuries, and losing long term custody of their children. Early intervention may help to prevent these events.

It is the duty of front line doctors to refer all children with suspected non-accidental injury. Failing to do so is a form of professional negligence. In the United States failing to report suspected child abuse is a federal offence punishable by imprisonment.

\section{Role of the consultant paediatrician}

It is vital to the interests of the child, the parents, and all the professionals concerned that the paediatrician reaches a correct and definite diagnosis in as many cases as possible. (This duty should not be delegated to junior staff.) If the paediatrician sits on the fence he or she will not only be in an uncomfortable position but also be doing a disservice to the child and the parents. If the paediatrician finds it impossible to decide initially he or she should admit the child to hospital while investigations continue. Few cases are uncertain after a thorough multidisciplinary investigation. The paediatrician should remember that he or she is being asked to commit himself or herself only on the balance of probabilities - not beyond all reasonable doubt. If the paediatrician genuinely cannot decide between non-accidental injury and an innocent injury, he or she should return an open verdict and decisions should be guided by the psychosocial assessment.

I thank the medical illustration units at the Royal Victoria Infirmary and Newcastle General Hospital for their help, particularly Mr Peter Grenecis, medical photographer at Dryburn Hospital. 